\title{
CDISC Protocol Subject Allocation Response Terminology
}

National Cancer Institute

\section{Source}

National Cancer Institute. CDISC Protocol Subject Allocation Response Terminology. NCI Thesaurus. Code C147068.

Terminology associated with the subject allocation response codelist of the Clinical Data Interchange Standards Consortium (CDISC) protocol entities. 